10,13

\title{
Влияние поверхности и границ раздела на продольный тепловой транспорт в слоистых тонкопленочных структурах $\mathrm{Si} / \mathrm{Ge}$
}

\author{
(ㄷ А.Л. Хомец ${ }^{1}$, И.И. Холяво ${ }^{1}$, И.В. Сафрронов ${ }^{2}$, А.Б. Филонов ${ }^{1}$, Д.Б. Мигас ${ }^{1,3}$ \\ ${ }^{1}$ Белорусский государственный университет информатики и радиоэлектроники, \\ Минск, Беларусь \\ ${ }^{2}$ Белорусский государственный университет, \\ Минск, Беларусь \\ ${ }^{3}$ Национальный исследовательский ядерный университет „МИФИ“, \\ Москва, Россия \\ E-mail: migas@bsuir.by
}

Поступила в Редакцию 17 декабря 2021 г.

В окончательной редакции 24 декабря 2021 г.

Принята к публикации 25 декабря 2021 г.

\begin{abstract}
Одним из основных подходов повышения термоэлектрической эффективности материалов является понижение их теплопроводности, в связи с чем в низкоразмерных структурах важную роль приобретает поверхность и возможные границы раздела. Проведено исследование продольной фононной теплопроводности в многослойных тонкопленочных структурах $\mathrm{Si} / \mathrm{Ge}$, имеющих резкие границы раздела и $(100),(110)$, (111) кристаллографические ориентации, в зависимости от числа периодов $\mathrm{Si} / \mathrm{Ge}$ (или толщины пленки) в сравнении с пленками Ge эквивалентной толщины методом неравновесной молекулярной динамики при $300 \mathrm{~K}$. Показано, что при уменьшении толщины слоистой пленки $\mathrm{Si} / \mathrm{Ge}$ c $\sim 50$ до $1 \mathrm{~nm}$ и распространении теплового потока вдоль направления [110] имеет место существенное фонон-поверхностное рассеяние для ориентации (100), что приводит к снижению фононной теплопроводности почти в 4 раза (с 19.1 до $5.12 \mathrm{~W} /(\mathrm{m} \cdot \mathrm{K}))$ и к незначительному ее изменению $(\sim 22 \pm 1 \mathrm{~W} /(\mathrm{m} \cdot \mathrm{K}))$ для ориентаций $(110)$ и $(111)$. $\mathrm{B}$ случае пленок Ge эквивалентной толщины установлено качественное и количественное соответствие с результатами для пленок $\mathrm{Si} / \mathrm{Ge}$, указывая на то, что рассеяние фононов на границе раздела $\mathrm{Si} / \mathrm{Ge}$ балансируется добавленными слоями $\mathrm{Si}$ с более высокой теплопроводностью.
\end{abstract}

Ключевые слова: фононная теплопроводность, тонкие пленки, слоистые структуры, кремний и германий, молекулярная динамика.

DOI: $10.21883 /$ FTT.2022.05.52338.257

\section{1. Введение}

В последнее десятилетие активно ведется поиск новых материалов для термоэлектрических применений. Важнейшим недостатком термоэлектрических материалов является их низкая эффективность и как следствие низкий КПД устройств, разработанных на их основе. Эффективность термоэлектрических материалов выражается с помощью безразмерного коэффициента $Z T$, который определяется как $S^{2} \sigma T /\left(\kappa_{\mathrm{L}}+\kappa_{\mathrm{e}}\right)$, где $S, \sigma, T$ и $\kappa_{\mathrm{L}}, \kappa_{\mathrm{e}}-$ соответственно коэффициенты термоэдс (Зеебека), электропроводности, рабочая температура и коэффициенты теплопроводности (фононная/решеточная и электронная составляющие). На данный момент одними из самых эффективных термоэлектрических материалов являются соединения на основе $\mathrm{Te}, \mathrm{Bi}$ и $\mathrm{Sb}, \mathrm{ZT}$ которых, для $p$ и $n$-типа материала, варьируется в диапазоне от $\sim 1$ до 2.5 [1-6]. А для соединения $\mathrm{SnSe}$ обнаружено одно из самых высоких значений $Z T$, равное $\sim 2.6$ при $923 \mathrm{~K}$ [7].

В настоящее время большое внимание уделяется $\mathrm{Si}$ и соединениям на его основе, так как он имеет ряд преимуществ: отработанная кремниевая технология, позволяющая создавать структуры с большим соотношением эффективность/стоимость, низкая токсичность по сравнению с традиционными термоэлектрическими материалами и широкий температурный диапазон работы термоэлектрических устройств. Однако, Si из-за слишком высокого коэффициента теплопроводности в $140 \mathrm{~W} /(\mathrm{m} \cdot \mathrm{K})$ при $300 \mathrm{~K}$ обладает достаточно низким коэффициентом $Z T \sim 0.01$ при $300 \mathrm{~K}$ [8]. В отличие от $\mathrm{Si}, \mathrm{Ge}$ обладает существенно меньшим значением коэффициента теплопроводности $(\sim 55 \mathrm{~W} /(\mathrm{m} \cdot \mathrm{K}))[9,10]$. Также установлено, что термоэлектрическая добротность $Z T$ для соединений на основе $\mathrm{Si}, \mathrm{SiGe}$ сплавов и сильно легированных $\mathrm{Si}$ материалов лежит в диапазоне от $\sim 0.3$ до 1.5 [11-16]. Для соединений $\mathrm{Si}$ и $\mathrm{Ge}$ самое высокое значение $Z T=1.84$ демонстрирует $\mathrm{SiGe}$ сплав $n$-типа [17]. Для достижения КПД в 20-30\% и начала массового применения устройств на основе термоэлектрических материалов значение $Z T$ должно составлять 3-4 [18].

Один из ключевых подходов для увеличения $Z T$ заключается в уменьшении фононной составляющей теплопроводности материала. Для этого используются полупроводниковые структуры пониженной размерности, к которым относятся тонкие пленки или нанопроволоки [19]. Например, в тонких пленках Si теплопроводность сильно зависит от их толщины и может 
снижаться за счет интенсивного фонон-поверхностного рассеяния до значений ниже $10 \mathrm{~W} /(\mathrm{m} \cdot \mathrm{K})$ при продольном тепловом транспорте [20], а в случае высокой дефектности теплопроводность может составлять $4.7 \mathrm{~W} /(\mathrm{m} \cdot \mathrm{K})$, при этом $Z T$ такой структуры достигает 0.2 при $360 \mathrm{~K}$ [21]. В экспериментальных работах также показано значительное снижение теплопроводности в сверхрешетках на основе $\mathrm{Si}$ и $\mathrm{Ge}$ по сравнению их с объемными аналогами из-за появления дополнительного фонон-интерфейсного рассеяния на границах раздела $\mathrm{Si} / \mathrm{Ge}$ [22-25]. В частности, теплопроводность в $\mathrm{Si} / \mathrm{Ge}$ сверхрешетках в зависимости от толщины слоев может снижаться до $\sim 3 \mathrm{~W} /(\mathrm{m} \cdot \mathrm{K})$ при $300 \mathrm{~K}$ [23]. В теоретических работах для этих же объектов основные усилия были направлены на исследование теплопроводности в зависимости от длины периода в симметричных периодических [26-33] и несимметричных апериодических сверхрешетках [34], от структуры границ раздела (учет шероховатости и/или взаимодиффузии [26,27,29,30,33]), a также температуры $[29,30,33]$. Однако практически нет работ, в которых исследовалась теплопроводность в тонких сверхрешетках, т. е. слоистых пленках с небольшим числом периодов. Такие сообщения были для поперечной теплопроводности, оцененной методом неравновесной молекулярной динамики, в симметричных слоистых пленках $\mathrm{Si} / \mathrm{Ge}$ [35] и при решении транспортного уравнения Больцмана для сверхрешеток $\mathrm{Si} / \mathrm{Ge}$ [36]. Не исключено, что дополнительным фактором понижения теплопроводности может быть выбор поверхностной ориентации сверхрешеток. Влияние этого эффекта на теплопроводность было рассмотрено ранее только для гомогенных тонких Si пленок [37-39], для которых была установлена наибольшая эффективность фонон-поверхностного рассеяния в случае поверхности $\mathrm{Si}(100)$. Следует отметить, что теоретически исследовались лишь тонкие пленки $\mathrm{Ge}(100)$, где выявили существенную анизотропию для продольной и поперечной составляющих теплопроводности в зависимости от толщины [40] и значительное влияние шероховатости поверхности на теплопроводность [41]. Данные для продольной теплопроводности слоистых пленок $\mathrm{Si} / \mathrm{Ge}$, а также тонких пленок $\mathrm{Ge}$, в зависимости от поверхностной ориентации и числа периодов или толщины отсутствуют. Таким образом, целью данной работы является исследование влияния поверхности и границ раздела в многослойных тонкопленочных структурах $\mathrm{Si} / \mathrm{Ge}$ на продольную фононную теплопроводность по сравнению с гомогенными пленками Ge для ориентаций (100), (110) и (111).

\section{2. Материалы и методы исследования}

Рассматривались (100)-, (110)- и (111)-ориентированные слоистые тонкопленочные структуры в виде симметричных гетероструктур $\mathrm{Si} / \mathrm{Ge}$ с резкими границами раздела, а также гомогенные пленки $\mathrm{Si}$ и Ge эквивалентной толщины. Число периодов варьировалось при неизменной толщине слоев $\mathrm{Si}$ и $\mathrm{Ge}$, которая выбиралась равной 4 моноатомным слоям для (100) и (110) ориентаций и 6 моноатомным слоям для (111) ориентации, чтобы избежать рассмотрения морфологий с островками на поверхности Ge [42]. Под периодом в работе понимается бислой $\mathrm{Si} / \mathrm{Ge}$. Использовались $p(2 \times 1), p(1 \times 1)$ и $p(2 \times 1)$ поверхностные реконструкции соответственно, как для гомогенных, так и слоистых пленок с (100), (110) и (111) ориентациями. Приготовление и визуализация всех структур осуществлялась с помощью программ Jmol [43] и OVITO [44]. Также для сравнения использовались данные, полученные для объемного массива $\mathrm{Si} / \mathrm{Ge}$ без поверхности и с различной ориентацией границ раздела $\mathrm{Si} / \mathrm{Ge}$.

Структурная оптимизация слоистых тонкопленочных структур $\mathrm{Si} / \mathrm{Ge}$ проводилась с помощью метода молекулярной статики, реализованного в программном пакете LAMMPS [45]. Минимизация полной энергии системы осуществлялась с учетом релаксации и оптимизации геометрии атомной структуры с переменной суперьячейкой. Использовались трехмерные периодические граничные условия с вакуумным зазором $\sim 2 \mathrm{~nm}$ над плоскостью укладки слоев для имитации тонкопленочной структуры. Межатомное взаимодействие для системы $\mathrm{Si}-\mathrm{Ge}$ описывалось с помощью потенциала Терсоффа [46]. Данный потенциал хорошо предсказывает термодинамические свойства объемного $\mathrm{Si}$ [47], $\mathrm{Ge}$ [47], твердых растворов $\mathrm{Si}-\mathrm{Ge}$ [46], и ранее был применен для моделирования теплопроводности пленок $\mathrm{Si} / \mathrm{Ge}[32,35,48,49]$.

Для моделирования фононной составляющей теплопроводности применялся метод неравновесной молекулярной динамики, реализованный в программном пакете LAMMPS [45]. Временной шаг задавался равным $1 \mathrm{fs}$. На первом этапе моделирования структуры приводились в термодинамическое равновесие с помощью изобарноизотермического и канонического ансамблей в течение $0.1 \mathrm{~ns}$ каждый при температуре $T=300 \mathrm{~K}$. На втором этапе моделирования для установления термодинамического равновесия использовался микроканонический ансамбль в течение $1 \mathrm{~ns}$. Для контроля и поддержания температуры в слоях применялись термостаты Ланжевена, при этом вводились соответствующие демпфирующие коэффициенты, влияющие на время релаксации во время колебаний, вследствие различия масс атомов. Для создания градиента температур в структурах устанавливались два термостата: холодный $(T=290 \mathrm{~K})$ и горячий $(T=310 \mathrm{~K})$ на расстоянии половины размера суперьячейки в направлении распространения теплового потока. Следует учитывать, что при использовании метода неравновесной молекулярной динамики рассчитываемый коэффициент теплопроводности зависит от размера суперъячейки, вдоль которой происходит распространение теплового потока, когда размер суперъячейки меньше или соизмерим со средней длинной свободного пробега фононов при данной температуре. Часто встречаемая недооценка (занижение) значений теплопроводности устраняется путем увеличения размера суперъячейки, что требует слишком больших вычислительных 


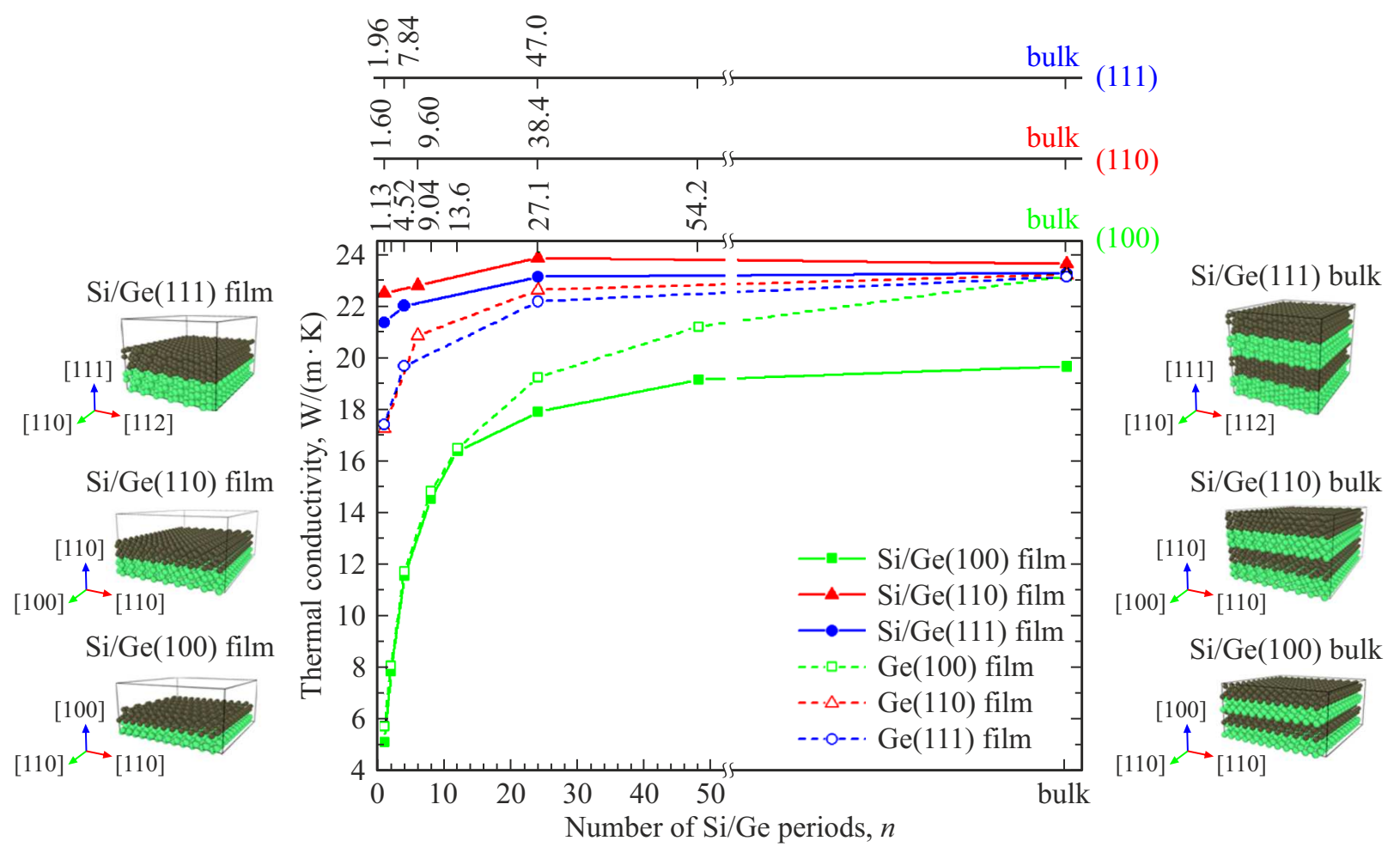

Продольная фононная теплопроводность вдоль направления [110] при $300 \mathrm{~K}$ и модели слоистых тонкопленочных структур $\mathrm{Si} / \mathrm{Ge}$ c (100), (110) и (111) ориентациями в зависимости от числа периодов. Соответствующие толщины для пленок Ge указаны на трех верхних осях в $\mathrm{nm}$.

затрат, однако, не оказывает существенного влияния на тенденции изменения теплопроводности. Считается, что для использования закона Фурье необходимо достичь режима линейного отклика между обратными величинами теплопроводности и длины суперъячейки. Согласно выводам авторов работы [47], например, для кристалла $\mathrm{Si}$ обратная величина теплопроводности становится линейно зависимой от обратной длины суперъячейки, когда последняя достигает размеров более $100 \mathrm{~nm}$. Учитывая, что средняя длина свободного пробега фононов (отношение теплопроводности к теплоемкости и средней скорости фононов) в кристалле Ge будет меньше, чем для $\mathrm{Si}(\sim 300 \mathrm{~nm}[50])$, то режим линейного отклика также должен выполняться в этом случае при выбранном размере суперъячейки. На основании этих выводов все многослойные тонкопленочные структуры $\mathrm{Si} / \mathrm{Ge}$, рассматриваемые в нашей работе, имели размер суперьячейки $\sim 100 \mathrm{~nm}$ в направлении распространения теплового потока вдоль слоев. Площадь поперечного сечения структур $\mathrm{Si} / \mathrm{Ge}$ задавалась $\sim 60 \mathrm{~nm}^{2}$.

Коэффициент фононной составляющей теплопроводности $\left(\kappa_{\mathrm{L}}\right)$ определялся из закона Фурье:

$$
\kappa_{\mathrm{L}}=-\frac{E}{2 S_{\mathrm{sec}} \cdot t \cdot(d T / d x)}
$$

где $E-$ перенесенная тепловая энергия; $2-$ коэффициент, связанный с распространением теплового потока в двух направлениях; $t$ - время моделирования;
$S_{\mathrm{sec}}$ - площадь поперечного сечения; $d T / d x-$ градиент температуры в направлении [110]. Вычисление коэффициента фононной составляющей теплопроводности проводилось через $2 \mathrm{~ns}$ моделирования.

\section{3. Результаты и обсуждение}

Вследствие кубической симметрии кристаллов $\mathrm{Si}$ и Ge направление [110] присутствует для пленок этих материалов с ориентациями (100), (110) и (111), что дает возможность сравнить вклад поверхности и границ раздела $\mathrm{Si} / \mathrm{Ge}$ на рассеяние фононов при распространении теплового потока вдоль этого направления для всех исследуемых наноструктур. На рисунке показана зависимость продольной теплопроводности (вдоль направления [110]) от числа периодов в многослойных тонкопленочных структурах $\mathrm{Si} / \mathrm{Ge}$, а также гомогенных пленках $\mathrm{Ge}$ эквивалентной толщины с (100), (110) и (111) поверхностями. Очевидно наличие ориентационного эффекта, который заключается в различной скорости насыщения продольной теплопроводности в рассматриваемых структурах. Для слоистых пленок $\mathrm{Si} / \mathrm{Ge}$ при увеличении числа периодов с 1 до 48 наблюдается значительное повышение (почти в 4 раза) продольной фононной теплопроводности для (100) ориентированных структур $\mathrm{Si} / \mathrm{Ge}$ с 5.12 до $19.1 \mathrm{~W} /(\mathrm{m} \cdot \mathrm{K})$, в то время как для структур $\mathrm{Si} / \mathrm{Ge}(110)$ и $\mathrm{Si} / \mathrm{Ge}(111)$ установлено лишь незначительное повышение соответственно с 22.5 
до $23.6 \mathrm{~W} /(\mathrm{m} \cdot \mathrm{K})$ и с 21.4 до $23.3 \mathrm{~W} /(\mathrm{m} \cdot \mathrm{K})$. Повышение продольной теплопроводности с увеличением числа периодов можно объяснить на основе концепции послойноограниченного и протяженного режимов теплопереноca [30], где показано, что с увеличением числа периодов повышается вклад в теплопроводность фононов, которые могут пересекать границы раздела и распространяться в вышележащих слоях (см. рисунок). С другой стороны также очевидна и роль поверхности в рассеянии фононов, так как только структуры $\mathrm{Si} / \mathrm{Ge}(100)$ характеризуются значительным уменьшением теплопроводности при уменьшении числа периодов (и количества границ раздела $\mathrm{Si} / \mathrm{Ge}$ и толщины пленки).

Аналогичные зависимости имеют место и для пленок $\mathrm{Ge}$ (см. рисунок). С увеличением их толщины (от $\sim 1 \mathrm{~nm}$ до массива) наблюдается повышение теплопроводности: с 5.72 до $23.1 \mathrm{~W} /(\mathrm{m} \cdot \mathrm{K})$, с 17.3 до $23.1 \mathrm{~W} /(\mathrm{m} \cdot \mathrm{K})$ и с 17.4 до $23.1 \mathrm{~W} /(\mathrm{m} \cdot \mathrm{K})$ соответственно для $(100),(110)$ и (111) ориентаций. Также как и для слоистых пленок $\mathrm{Si} / \mathrm{Ge}(100)$ в случае гомогенных пленок $\mathrm{Ge}(100)$ отмечается существенное (четырехкратное) повышение теплопроводности с увеличением толщины, что не наблюдается для (110) и (111) ориентаций.

Ранее подобная тенденция обнаружена для тонких пленок $\mathrm{Si}$ при изменении их толщины (10 nm и менее) вследствие перераспределения вкладов фонон-фононного и фонон-поверхностного рассеяний, а также эффекта фононного обеднения [51]. В случае продольного теплового транспорта в тонких пленках $\mathrm{Si}$ вдоль направления [110] отличаются не только скорости фононного рассеяния (обратная величина времени релаксации), но и групповые скорости фононов (пропорциональна теплопроводности) [38,39]. Авторами работы [39] было показано, что график частотной зависимости средневзвешенной по всем модам групповой скорости фононов для случая $\mathrm{Si}(110)$ лежит выше по сравнению с $\mathrm{Si}(100)$ и $\mathrm{Si}(111)$. Касательно рассеяния фононов, в работе [38] на основе анализа формы их изоэнергетических поверхностей для кристалла $\mathrm{Si}$ было продемонстрировано, что при продольном тепловом транспорте в тонких пленках $\mathrm{Si}$ максимальной рассеивающей способностью обладают (100) поверхности. Применительно к рассматриваемым слоистым структурам $\mathrm{Si} / \mathrm{Ge}$ и гомогенным пленкам Ge их продольная теплопроводность определятся как групповой скоростью, так и временем релаксации рассеяния фононов, которые являются наибольшими для ориентации (110) и наименьшими для ориентации (100), что и объясняет полученную зависимость на рисунке.

Учитывая, что теплопроводность объемного Ge $(\sim 55 \mathrm{~W} /(\mathrm{m} \cdot \mathrm{K})[9,10])$ ниже в $\sim 2.5$ раза, чем для $\mathrm{Si}$ $(\sim 140 \mathrm{~W} /(\mathrm{m} \cdot \mathrm{K})[52,53])$, то в первую очередь представляет интерес возможный выигрыш с точки зрения понижения теплопроводности слоистых тонкопленочных структур $\mathrm{Si} / \mathrm{Ge}$ по сравнению с гомогенными пленками Ge за счет границ раздела $\mathrm{Si} / \mathrm{Ge}$. В случае ориентации (100) такой выигрыш имеет место, в то время как для ориентаций (110) и (111) наблюдается обратная закономерность (см. рисунок). То есть фононинтерфейсное рассеяние в первом случае компенсирует вклад в общую теплопроводность более теплопроводящих слоев $\mathrm{Si}$. При этом только для ориентации (100) с увеличением числа периодов повышается разность в теплопроводности для слоистой тонкопленочной структуры $\mathrm{Si} / \mathrm{Ge}$ и гомогенной пленки $\mathrm{Ge}$ эквивалентной толщины с $\sim 10.5$ до $17.3 \%$ при одном периоде и массиве, соответственно. Это объясняется тем, что в случае слоистой пленки $\mathrm{Si} / \mathrm{Ge}(100)$ с увеличением числа границ раздела происходит более интенсивное рассеяние фононов по сравнению с гомогенной пленкой $\mathrm{Ge}$, тогда как для $\mathrm{Si} / \mathrm{Ge}(110)$ и $\mathrm{Si} / \mathrm{Ge}(111)$ границы раздела слабее рассеивают фононы и различия заметны лишь при малом числе периодов, когда дополнительное влияние оказывают свободные поверхности.

По сравнению с гомогенными пленками $\mathrm{Si}$ эквивалентной толщины слоистые пленки $\mathrm{Si} / \mathrm{Ge}$ имеют более низкую теплопроводность. При увеличении толщины пленки $\mathrm{Si}(100)$ с 1.09 до $52.8 \mathrm{~nm}$ (эквивалентная толщина структуры $\mathrm{Si} / \mathrm{Ge}$ увеличивается соответственно с 1 до 48 периодов) происходит увеличение теплопроводности с 10.9 до $34.3 \mathrm{~W} /(\mathrm{m} \cdot \mathrm{K})$, что на $\sim 45-50 \%$ выше, чем у эквивалентной по толщине тонкопленочной структуры $\mathrm{Si} / \mathrm{Ge}$. Для ориентаций $\mathrm{Si}(110)$ и $\mathrm{Si}(111)$ наблюдается аналогичная тенденция и значения фононной теплопроводности для тонких пленок с данными ориентациями на $\sim 45 \%$ выше, чем в слоистых тонкопленочных структурах $\mathrm{Si} / \mathrm{Ge}$ эквивалентной толщины (не показано на рисунке).

Для объемной сверхрешетки $\mathrm{Si} / \mathrm{Ge}$ (массива) хорошо видна разница в значениях теплопроводности в зависимости от ориентации границ раздела, особенно для $\mathrm{Si} / \mathrm{Ge}(100)$. То есть, несмотря на отсутствие поверхностей, численные значения теплопроводности не сходятся в одной точке в отличие от объемного $\mathrm{Ge}$ (см. рисунок). Это выявляет существенную роль границ раздела $\mathrm{Si} / \mathrm{Ge}$ с такой ориентацией плоскостей, которая определяет наиболее интенсивное рассеяние фононов. Последнее связано с тем, что при продольном тепловом транспорте направления движения фононов не совпадают с направлением их преимущественного рассеяния. В связи с этим, как видно из рисунка, объемные сверхрешетки $\mathrm{Si} / \mathrm{Ge}$ с (110) и (111) ориентациями границ раздела не имеют преимущества по сравнению с монокристаллами $\mathrm{Ge}$.

Также проведены дополнительные расчеты для слоистых тонкопленочных структур $\mathrm{Si} / \mathrm{Ge}$ с добавленными поверхностными слоями $\mathrm{Si}$ или $\mathrm{Ge}$, чтобы получить обе терминирующие поверхности гетероструктуры из одного и того же материала. Для ориентации (100) не обнаружено практически никаких существенных изменений зависимости теплопроводности от числа периодов (толщины), представленной на рисунке, как для случая с поверхностными слоями $\mathrm{Si}$, так и $\mathrm{Ge}$. Для ориентаций (110) и (111) обнаружено понижение (или повышение) теплопроводности на $3-4 \mathrm{~W} /(\mathrm{m} \cdot \mathrm{K})$ 
при толщинах $\sim 10 \mathrm{~nm}$, если оба поверхностных слоя образованы атомами $\mathrm{Ge}$ (или $\mathrm{Si}$ ). Однако этот эффект очень быстро нивелируется при толщинах $\sim 32-39 \mathrm{~nm}$ (на рисунке не отображено). Таким образом, для тонкопленочных структур $\mathrm{Si} / \mathrm{Ge}(110)$ и $\mathrm{Si} / \mathrm{Ge}(111)$ также возможно дополнительное изменение их теплопроводность путем выбора материала терминирующих поверхностей, но только при толщинах пленок менее $\sim 10 \mathrm{~nm}$.

\section{4. Заключение}

Результаты расчетов продольной фононной теплопроводности в гомогенных пленках $\mathrm{Ge}$ и слоистых тонкопленочных структурах $\mathrm{Si} / \mathrm{Ge}$ c (100), (110) и (111) ориентациями в зависимости от их толщины, полученных с помощью метода неравновесной молекулярной динамики, показали схожие зависимости. Пленки с ориентациями (100) по сравнению с (110) и (111) ориентациями характеризуются наименьшими значениями теплопроводности и существенными ее изменениями от толщины, что свидетельствует о наличии ориентационного эффекта при рассмотрении теплового потока вдоль направления [110]. Этот эффект обусловлен главным образом поверхностью, а границы раздела в случае пленок $\mathrm{Si} / \mathrm{Ge}$ не так эффективно рассеивают фононы. Следует отметить, что количественно данные для пленок $\mathrm{Si} / \mathrm{Ge}$ и $\mathrm{Ge}$ эквивалентной толщины также сопоставимы. Это указывает на то, что по сравнению с пленками Ge в пленках $\mathrm{Si} / \mathrm{Ge}$ фонон-интерфейсное рассеяние уравновешивает, либо не уравновешивает вклад более теплопроводящих слоев $\mathrm{Si}$. Только в случае пленок $\mathrm{Si} / \mathrm{Ge}(100)$ обнаружено понижение теплопроводности по сравнению с гомогенными пленками $\mathrm{Ge}(100)$ на $\sim 10.5-17.3 \%$ по мере увеличения числа периодов, т.е. в диапазоне сотен nm возможно значительное понижение теплопроводности за счет дополнительного рассеяния на границах раздела. Полученные данные демонстрируют, что изменяя число периодов $\mathrm{Si} / \mathrm{Ge}$, и выбирая соответствующую кристаллографическую ориентацию можно изменять величину фононной теплопроводности слоистых тонкопленочных структур $\mathrm{Si} / \mathrm{Ge}$. Однако для более точной оценки эффективности термоэлектрических устройств на основе рассматриваемых наноструктур необходимо проведение дополнительных исследований возможного изменения фактора мощности, влияния температуры на теплопроводность, а также исследовать их поперечную теплопроводность и теплопроводность вдоль других кристаллографических направлений, что и является предметом дальнейших исследований.

\section{Благодарности}

Авторы выражают благодарность профессору Н.А. Поклонскому (Белорусский государственный университет) за плодотворные обсуждения.

\section{Финансирование работы}

Работа была выполнена в рамках научной государственной программы Республики Беларусь „Материаловедение, новые материалы и технологии“.

\section{Конфликт интересов}

Авторы заявляют, что у них нет конфликта интересов

\section{Список литературы}

[1] J.P. Heremans, V. Jovovic, E.S. Toberer, A. Saramat, K. Kurosaki, A. Charoenphakdee, S. Yamanaka. Science 321, 5888, 554 (2008).

[2] L. Hu, T. Zhu, X. Liu, X. Zhao. Adv. Func. Mater. 24, 33, 5211 (2014).

[3] Y. Pei, A. LaLonde, S. Iwanaga, G. Jeffrey Snyder. Energy Environ. Sci. 4, 6, 2085 (2011).

[4] A.D. LaLonde, Y. Pei, G. Jeffrey Snyder. Energy Environ. Sci. 4, 6, 2090 (2011).

[5] Pierre F.P. Poudeu Dr., J. D’Angelo, A.D. Downey, J.L. Short, T.P. Hogan, M.G. Kanatzidis. Angewandte Chem. Int. Ed. 45, 23, 3835 (2006).

[6] G. Tan, F. Shi, S. Hao, Li-Dong Zhao, H. Chi, X. Zhang, C. Uher, C. Wolverton, Vinayak P. Dravid Mercouri G. Kanatzidis. Nature Commun. 7, 12167 (2016).

[7] Li-Dong Zhao, Shih-Han Lo, Y. Zhang, H. Sun, G. Tan, C. Uher, C. Wolverton, V.P. Dravid, M.G. Kanatzidis. Nature 508, 373 (2014).

[8] T.H. Geballe, G.W. Hill. Phys. Rev. 98, 4, 940 (1955).

[9] A.F. Ioffe. Can. J. Phys. 34 (12A), 1342 (1956).

[10] V.I. Ozhogin, A.V. Inyushkin, A.N. Taldenkov, A.V. Tikhomirov, G.É. Popov, E. Haller, K. Itoh. J. Exp. Theor. Phys. Lett. 63, 490 (1996).

[11] J.P. Dismukes, L. Ekstrom, E.F. Steigmeier, I. Kudman, D.S. Beers. J. Appl. Phys. 35, 10, 2899 (1964).

[12] V. Kessler, D. Gautam, T. Hülser, M. Spree, R. Theismann, M. Winterer, H. Wiggers, G. Schierning, R. Schmechel. Adv. Eng. Mater. 15, 5, 379 (2012).

[13] C.B. Vining, W. Laskow, J.O. Hanson, R.R. Van der Beck, P.D. Gorsuch. J. Appl. Phys. 69, 8, 4333 (1991).

[14] X.W. Wang, H. Lee, Y.C. Lan, G.H. Zhu, G. Joshi, D.Z. Wang, J. Yang, A.J. Muto, M.Y. Tang, J. Klatsky, S. Song, M.S. Dresselhaus, G. Chen, Z.F. Ren. Appl. Phys. Lett. 93, 19, 193121 (2008).

[15] S. Bathula, M. Jayasimhadri, N. Singh, A.K. Srivastva, J. Pulikkotil, A. Dhar, R.C. Budhani. Appl. Phys. Lett. 101, 21, 213902 (2012).

[16] A. Yusufu, K. Kurosaki, Y. Miyazaki, M. Ishimaru, A. Kosuga, Y. Ohishi, H. Muta, S. Yamanaka. Nanoscale 6, 22, 13921 (2014).

[17] R. Basu, S. Bhattacharya, R. Bhatt, M. Roy, S. Ahmad, A. Singh, N. Navaneethan, Y. Hayakawa, D.K. Aswai, S.K. Gupta. J. Mater. Chem. A 2, 19, 6922 (2014).

[18] А.Ф. Иоффе. Полупроводниковые термоэлементы. Изд-во AH CCCP, M, (1956). 103 c.

[19] J.A. Pérez-Taborda, O. Caballero-Calero, M. Martín-González. New Research on Silicon - Structure, Properties, Technology. InTechOpen. London. (2017). P. 183. 
[20] C. Jeong, S. Datta, M. Lundstorm. J. Appl. Phys. 111, 9, 093708 (2012).

[21] N.S. Bennett, N.M. Wight, S.R. Popuri, Jan-Willem G. Bos. Nano Energy 16, 350 (2015).

[22] S.-M. Lee, David G. Cahill, R. Venkatasubramanian. Appl. Phys. Lett. 70, 22, 2957 (1997)

[23] T. Borca-Tasciuc, W. Liu, J. Liu, T. Zeng, David W. Song, C.D. Moore, G. Chen, Kang L. Wang, M.S. Goorsky, T. Radetic, R. Gronsky, T. Koga, M.S. Dresselhaus. Superlat. Microstruct. 28, 3, 199 (2000).

[24] W.L. Liu, T. Borca-Tasciuc, G. Chen, J.L. Liu, K.L. Wang. J. Nanosci. Nanotechnology 1, 1, 39 (2001)

[25] S. Chakraborty, C. A. Kleint, A. Heinrich, C.M. Schneider, J. Schumann, M. Falke, S. Teichert. Appl. Phys. Lett. 83, 20, 4184 (2003).

[26] E.S. Landry, A.J.H. Mc Gaughey. Phys. Rev. B 79, 7, 075316 (2009).

[27] J. Grag, G. Chen. Phys. Rev. B 87, 14, 140302 (2013).

[28] Keng-Hua Lin, A. Strachan. Phys. Rev. B 87, 11, 115302 (2013).

[29] Z. Aksamija, I. Knezevic. Phys. Rev. B 88, 15, 155318 (2013).

[30] K. Kothari, M. Maldovan. Sci. Rep. 7, 5625 (2017).

[31] H. Dong, B. Wen, Y. Zhang, R. Melnik. RSC Advances 7, 48, 29959 (2017).

[32] A. Kandemir, A. Ozden, T. Cagin, C. Sevik. Sci. Technol. Adv. Mater. 18, 1, 187 (2017).

[33] G.P. Srivastava, Lorwerth O. Thomas. Nanomaterials 10, 673 (2020).

[34] J. Yan, H. Wei, H. Xie, X. Gu, H. Bao. ES Energy Environment 8, 56 (2020)

[35] V. Samvedi, V. Tomar. J. Appl. Phys. 105, 1, 013541 (2009).

[36] A. Malhotra, K. Kothari, M. Maldovan. J. Appl. Phys. 125, 4, 044304 (2019).

[37] P. Heino. Eur. Phys. J. B 60, 171 (2007).

[38] Z. Aksamija, I. Knezevic. Phys. Rev. B 82, 4, 045319 (2010).

[39] H. Karamitaheri, N. Neophytou, H. Kosina. J. Appl. Phys. 113, 20, 204305 (2013).

[40] Z. Xingli, W. Xiande. Comput. Mater. Sci. 123, 40 (2016).

[41] Z.H. Wang, M.J. Ni. Heat Mass Transfer 47, 449 (2011).

[42] B. Voigtländer. Surface Sci. Rep. 43, 5-8, 127 (2001).

[43] Jmol: an open-source Java viecer for chemical structures in 3D. http://www.jmol.org/

[44] A. Stukowski. Mod. Simul. Mater. Sci. Eng. 18, 015012 (2009).

[45] S. Plimpton. J. Comp. Phys. 117, 1 (1995).

[46] J. Tersoff. Phys. Rev. B 39, 8, 5566 (1989).

[47] Y. He, I. Savić, D. Donadio, G. Galli. Phys. Chem. Chem. Phys. 14, 47, 16209 (2012).

[48] Z. Wang. Mater. Today Commun. 22, 100822 (2020).

[49] A. Giri, Jeffrey L. Braun, Patrick E. Hopkins. J. Appl. Phys. 119, 23, 235305 (2016).

[50] Y.S. Ju, K.E. Goodson. Appl. Phys. Lett. 74, 20, 3005 (1999).

[51] X. Wang, B. Huang. Sci. Rep. 4, 6399 (2014).

[52] H.R. Shanks, P.D. Maycock, P.H. Sidles, G.C. Danielson. Phys. Rev. 130, 5, 1743 (1963).

[53] W.S. Capinski, H.J. Maris. E. Bauser, I. Silier, M. Asen-Palmer, T. Ruf, M. Cardona, E. Gmelin. Appl. Phys. Lett. 71, 15, 2109 (1997).

Редактор К.В. Емиев 